\title{
Modeling Vaccinations, Virus Variants and Lockdown: Early guidance for Sars-Cov-2 health policies in India *
}

\author{
Computational Decision Science Laboratory \\ Yi Zhang and Sanjiv Kapoor ${ }^{\dagger}$ \\ Illinois Institute of Technology, Chicago, USA.
}

\begin{abstract}
In this paper we consider long range models that help in predicting the impact of virus transmission over a period of a few months, . This is applied in the context of the SARS-CoV2 virus for India. Long range models are useful for preemptive policy actions. Our models utilize a dynamic transmission factor, dependent on the population's knowledge and tendency to be careful when the case load of virus transmission rises. Our model incorporates the state of vaccination and the virus variants that form part of the transmission dynamics.

We also present the impact of lockdown policies and illustrate the advantage of adopting partial lockdown policies in the early period of 2022 .
\end{abstract}

\section{Introduction}

The SARS-COV2 virus has been plaguing the world for 2 years, compounding the original problem with multiple variants. Containment is key to preventing the spread and also to contain variants. Accuracy in models that predict the growth in infections are very important. While short term analysis of a few weeks is typically more accurate, long range models are important in planning. In this paper we consider the impact of lockdown policies, vaccinations along with the vaccine-resistant virus variants.

Effective public health policy requires timely enhance social distancing guidelines; as such it is critical to develop early-warning systems that would predict the rise of the virus. These warning systems would include parameters that reflect the relaxing of norms related to masking, enhanced mobility etc. that reflect an influx of susceptible into the population. Since the incubation period of the disease and the spread in asymptomatic population, especially vaccinated, results in delayed impact on the progression of the disease, long term modeling of the spread of the virus is critical. While it is anticipated that lockdown removal will result in resurgence of the virus, lomg termimpact of the policies on the resurgence requires appropriate modeling.

This paper deals with an analysis of the spread of infection as more population emerges from lockdown and adopts lax social norms with respect to social distancing. Impact of relaxed norms are apparent in the multiple waves that have afflicted countries around the world. We construct a model that reflects the vaccination status of the population as well as incorporate virus variants accounting for the efficacy

${ }^{*}$ Research sponsored by NSF. grant No. 2028274.

Corresponding author, kapoor@iit.edu 
of the vaccines. The analysis is based on epidemic spread models that utilize compartment models [3, 5] (originally from Kermack and McKendrick [6, 7, 8]). A key differentiation in our work is the use of dynamic social distancing factor in the model, termed the SIR-SD model introduced in [19]. The role of reducing contacts to reduce transmission is well studied [18, 4, 9] as and more recent modeling efforts have focused also on mobility [2], amongst other factors [11, 15, 10, 16, 1, 13, 14, 12, 17].

We consider a version of the compartment models that include compartment reflecting the various states of the population including vaccinated population, population under lockdown etc. As mentioned above, we also use a social distancing factor to modify the virus transmission rate that reflects the behavior of the population as the number of infection cases rises and reporting of the infections, as well as government mandates, becomes more prevalent. As introduced earlier in [19] the transmission of the virus incorporates a factor that is inversely dependent on the number of cumulative infections incorporated by a time-dependent susceptible population size.

This model has been utilized for multiple months starting from June 2021 in order to provide predictive scenarios multiple months in advance. We calibrated our model using the second wave of infections in India in April 2021. The wave had come as a surprise to policy makers and we determined that a long range model was important to ensure that the infection growth was contained as lockdown was removed and vaccination rates grew. We assessed the growth of susceptible population, $H$, that led to the second wave and based future projections on the relative rate at which the population entered the susceptible set, as a result of their social distancing behavior and government policies. We considered three rates: a fast rate of release where the susceptible population $H$ was released in 30 days; a medium rate of release where the susceptible population $H$ was released in 45 days and a slower rate of release of $H$ population count over 60 days. We illustrate the succession of projections for the fast release rate (30 day release) in Figure 1. The projections range is bounded by an optimistic and pessimistic scenario. The pessimistic scenario is when the addition to susceptible population is not bounded as additional elements of the population may join to ensure the release rate. In the optimistic scenario the number of population released is bounded by the numbers released into the susceptible population during the second wave in April. The low rate of growth of infections could possibly be attributed to slow release from lockdown and enhance vaccination rates.

Previous versions of this document have been indicated to Dr. V.K. Paul (Niti Ayog, GOI) to provide monthly updated projections from June 2021. The projections were accompanied by data on social distancing and mask usage from IHME as requested by Dr. Paul. However we did not use these metrics as part of our model as currently this data is based on surveys and was typically delayed.

The combination of social distancing and mobility over the time period is illustrated in Figure 2 which illustrates the decreases in social distancing and Covid prevention metrics. While the cases incidences were kept limited till November the impact of waning vaccine efficacy likely had an impact on the cases incidences in December.

\section{Methods}

We use a mechanistic model as illustrated in Figure 3. Apart from standard compartments representing the susceptible $S$, Exposed $E$, Infected (confirmed via testing) $I$, Undetected Symptomatic and Asymptomatic $A$, we include hospitalized $I_{H}$ and home treated $I_{N}$, along with dead $D$ and recovered $R$. 

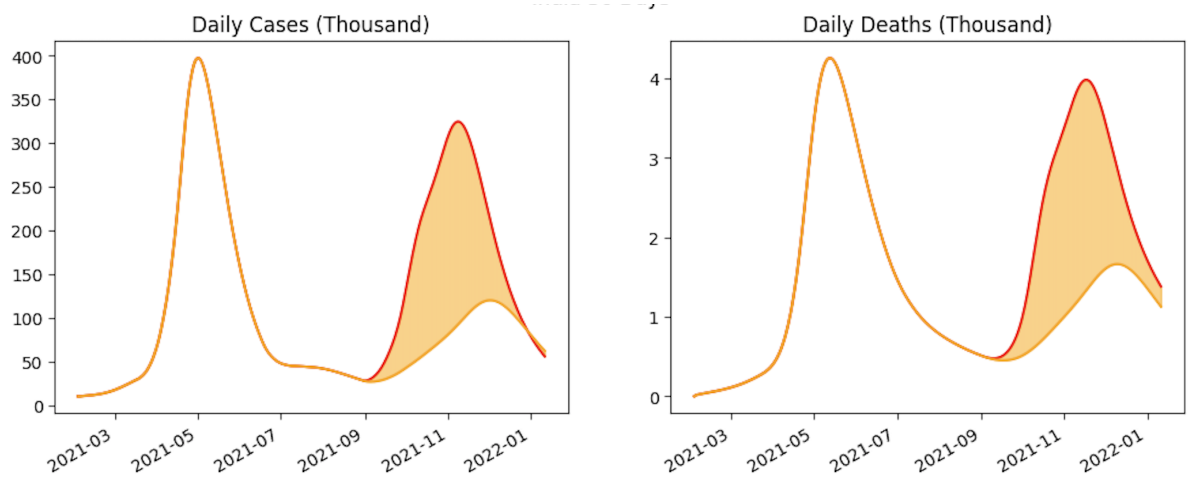

(a) August Simulations
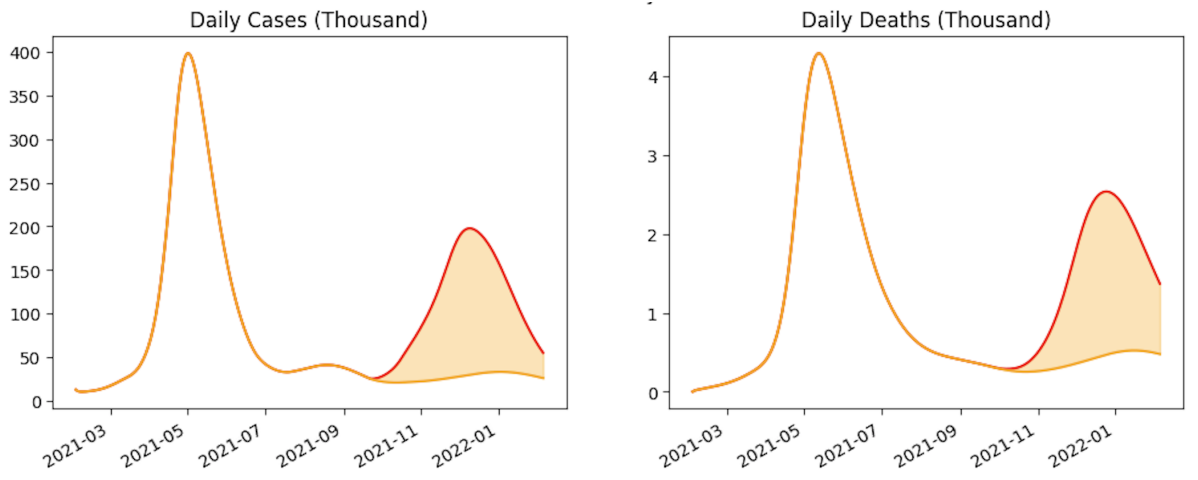

(b) September Simulations
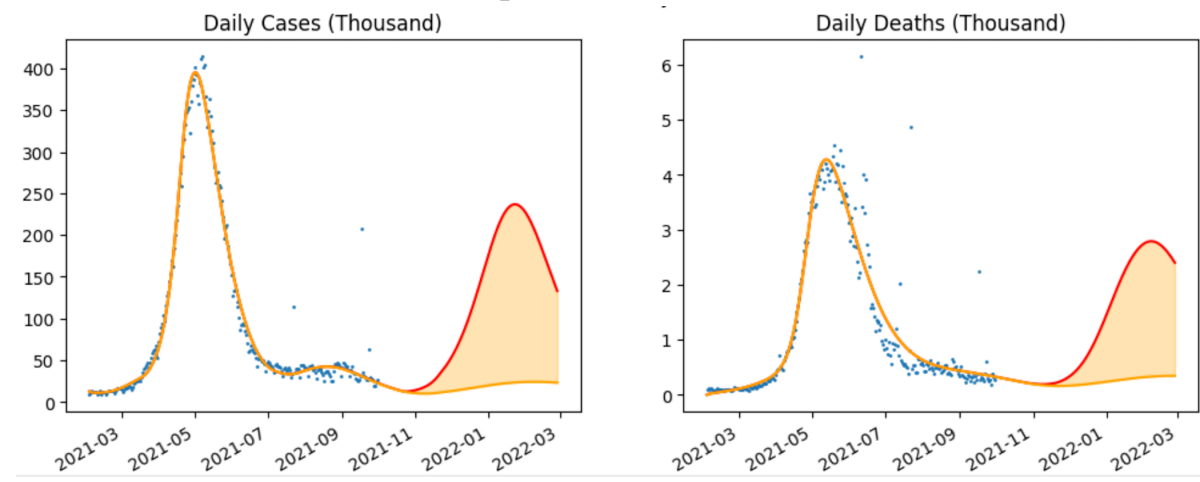

(c) October Simulations
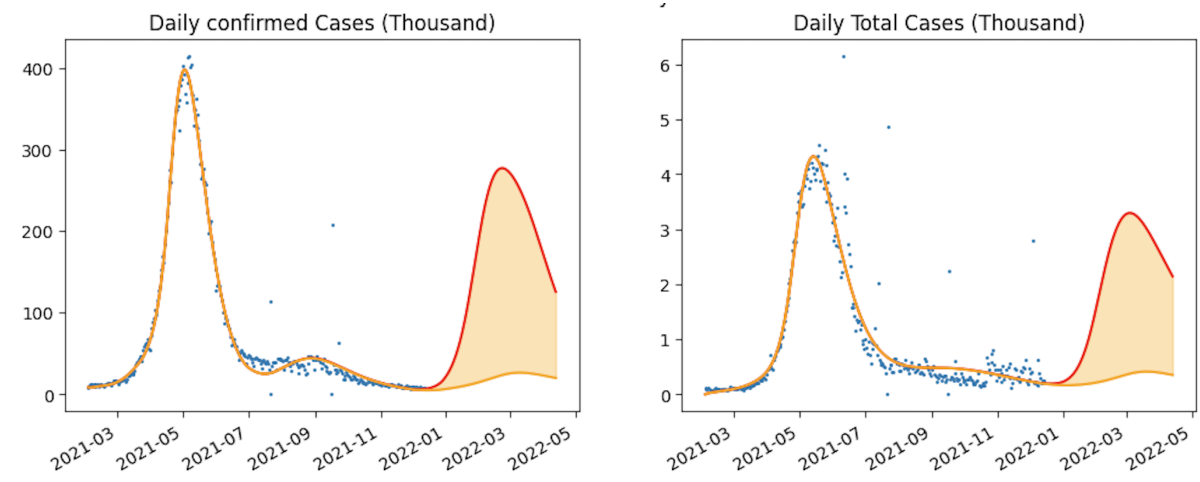

(c) November Simulations

3

Figure 1: Monthly sequence of projections for the case of fast release rate. The peak case load varied according to the current infection trends and successively delayed by almost a month. 

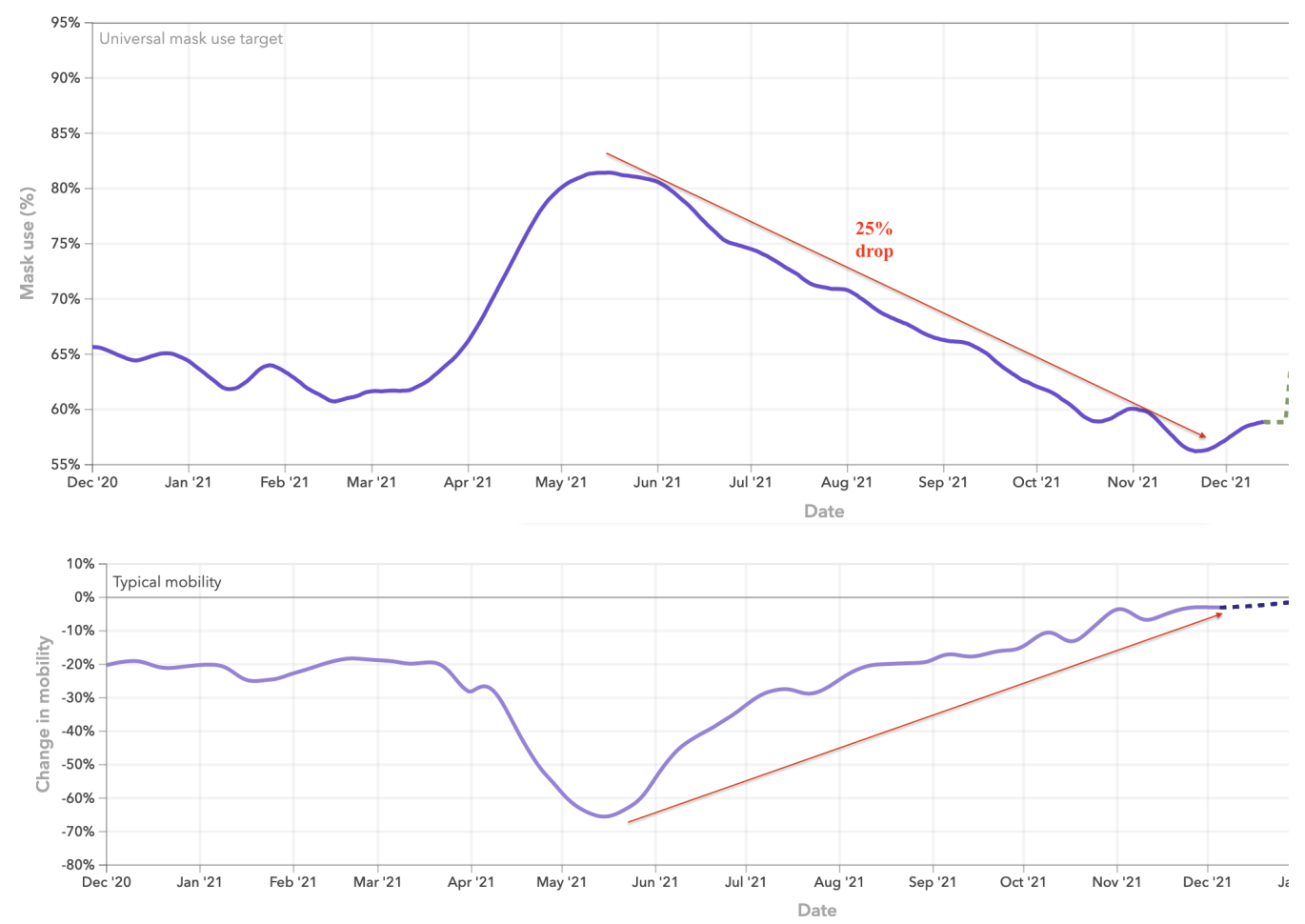

Figure 2: IHME: survey of masks usage and mobility

Each of these compartments are replicated for various types of virus variants to understand the impact of each type of virus variant. In the context of the Omicron virus in addition to the base variant, the Delta variant, these compartments are indexed by superscript $O$. Additionally we have a population that is on lockdown, represented by $H$. The various stages of vaccinations are represented by $H_{1}$ : first shot of vaccination; $\mathrm{H}_{2}$ : second shot of vaccination; $\mathrm{H}_{3}$ : third shot of vaccination; $\mathrm{H}_{4}$ : vaccinated population after 4 months. Vaccination efficacy has been know to drop by $50 \%$ after 4 months and $H_{4}$ model this.

A release from lockdown transfers population from the corresponding lockdown compartments to the susceptible population, while lockdown policies transfer susceptible back to the $H$ compartments.

\subsection{The Compartment Model}

$$
\begin{aligned}
\frac{d H_{0}}{d t}=-v_{1} H_{0}-\kappa^{t} \cdot \frac{H_{0}}{\hat{H}} ; & \frac{d H_{1}}{d t}=v_{1} H_{0}-v_{2} H 1-\kappa^{t} \cdot \frac{H_{1}}{\hat{H}} ; \\
\frac{d H_{2}}{d t}=v_{2} H_{1}-v_{3} H_{2}-\kappa^{t} \cdot \frac{H_{2}}{\hat{H}} ; & \frac{d H_{3}}{d t}=v_{3} H_{2}-\kappa^{t} \cdot \frac{H_{3}}{\hat{H}} ; \\
\frac{d S_{0}}{d t}=\kappa^{t} \cdot \frac{H_{0}}{\hat{H}}-v_{1} S_{0}-\sum_{C} \frac{\beta_{0}^{C} S_{0}^{C} \hat{I}^{C}}{N} ; & \frac{d S_{1}}{d t}=\kappa^{t} \cdot \frac{H_{1}}{\hat{H}}+v_{1} S_{0}-v_{2} S_{1}-\sum_{C} \frac{\beta_{1}^{C} S_{1}^{C} \hat{I}^{C}}{N} ; \\
\frac{d S_{2}}{d t}=\kappa^{t} \cdot \frac{H_{2}}{\hat{H}}+v_{2} S_{1}-v_{3} S_{2}-\sum_{C} \frac{\beta_{2}^{C} S_{2}^{C} \hat{I}^{C}}{N} ; & \frac{d S_{3}}{d t}=\kappa^{t} \cdot \frac{H_{3}}{\hat{H}}+v_{3} S_{2}-\sum_{C} \frac{\beta_{3}^{C} S_{3}^{C} \hat{I}^{C}}{N} ;
\end{aligned}
$$




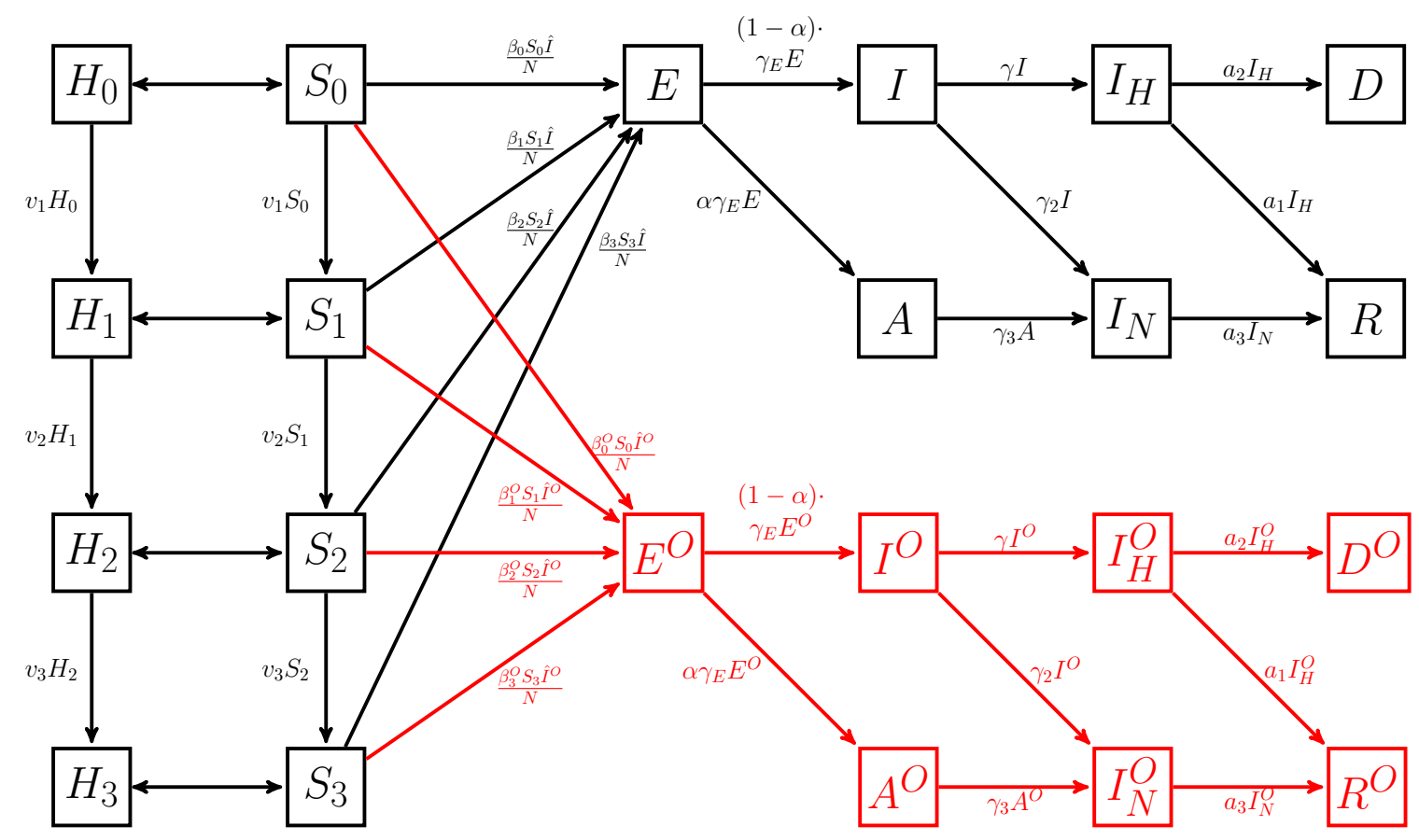

Figure 3: The SIR-SD-L Compartment Model with vaccination and two mutations of the virus; delta and omicron (red)

And $\forall C$,

$$
\begin{array}{rlrl}
\frac{d E^{C}}{d t} & =\frac{\hat{\beta S} S^{C} \cdot \hat{I}^{C}}{N}-\gamma_{E} E^{C} ; & \frac{d I^{C}}{d t}=(1-\alpha) \gamma_{E} E^{C}-\left(\gamma+\gamma_{2}\right) I^{C} \\
\frac{d A^{C}}{d t}=\alpha \gamma_{E} E^{C}-\gamma_{3} A^{C} ; & \frac{d I_{H}^{C}}{d t}=\gamma I^{C}-\left(a_{1}+a_{2}\right) I_{H}^{C} ; \\
\frac{d I_{N}^{C}}{d t}=\gamma_{2} I^{C}-a_{3} I_{N}^{C} ; & \frac{d D^{C}}{d t}=a_{2} I_{H}^{C} ; \\
\frac{d R^{C}}{d t}=a_{1} I_{H}^{C}+a_{3} I_{N}^{C} ; & &
\end{array}
$$

where $\hat{H}=H_{0}+H_{1}+H_{2}+H_{3}$, and $\hat{\beta S}{ }^{C}=\beta_{0}^{C} S_{0}+\beta_{1}^{C} S_{1}+\beta_{2}^{C} S_{2}+\beta_{3}^{C} S_{3}, \hat{I}^{C}=I^{C}+A^{C}$.

To model the impact of the virus on the population's behavior we utilize dynamic transmission factor $\beta$ [19]. The value of $\beta$ is based on the following generic transmission function that models the dynamics of population behavior (for further discussion see [19]:

$$
\beta=\beta_{0} \frac{1-c_{1}}{1-c_{1} \cdot \frac{S}{S_{0}}}
$$

where $S$ is the number of the current susceptible population and $S_{0}$ the original set of susceptible population. 
The function $\kappa^{t}$ varies dependent on whether the policies are in a release phase or in a lockdown phase. We use a linear function in our model:

- Release Phase: In the release phase, between time $t_{1}$ and $t_{2}$, the function $\kappa^{t}=h^{R}, t_{1} \leq t \leq t_{2}$ where $h^{R}$ is a positive constant and where $t_{1}$ and $t_{2}$ is the range of time over which the population enters into a susceptible state. The value of $h^{R}$ is determined by the release policies and adherence.

- Lockdown Phase: In the lockdown phase, between time $t_{3}$ and $t_{4}$, the function $\kappa^{t}=h^{L}, t_{3} \leq t \leq t_{4}$ where $h^{L}$ is a negative constant is the range of time over which the population withdraws into a state of lockdown. The value of $h^{L}$ is determined by the strength of the lockdown and adherence.

\section{Methodology}

Our method for determining parameters of the model used an optimization method to generate a best-fit, utilizing a loss function.

We let $G, D$ denote the simulated cumulative cases and deaths, $\bar{G}, \bar{D}$ are the corresponding reported data. We use an optimization methodology, starting with random initial choices of the parameters over defined ranges as well as a search over the space of release dates to achieve the best fit. The optimization method used is L-BGFS that converges to an optimum point, which due to the non-convex nature of the problem could possibly be a local optima. Sampling multiple starting points to find the best fit leads to reasonably accurate fitting as measured by $R^{2}$ values.

\subsection{First-Phase fitting}

In the first phase we fit the parameters related to the first variant of the virus. We consider the period from February up till June 10th, 2021 when the removal of lockdown began, as an effective release phase where the population behavior was relaxed with low mask usage and high mobility. While around mid April, 2021 there was a lockdown, we consider the entire period as an effective release period. The release of susceptible population was assumed to be a linear function with a starting date in a range of 1 week beginning on 15th March. These dates were sampled to compute the release function that resulted in the best fit.

The parameters we considered for fitting are $\beta, \gamma_{E}, \alpha, \gamma, \gamma_{2}, \gamma_{3}, a_{1}, a_{2}, a_{3}, h_{R}, t_{1}, t_{2}$ that are used to generate the series $G$ and $D$ according to the differential equations governing the model.

We use the following loss function.

$$
\begin{aligned}
& L_{1}\left(G, D, r_{a b}\right)= \\
& -\left[\theta_{1} R^{2}(G, \bar{G})+\theta_{2} R^{2}(d G, d \bar{G})+\theta_{3} R^{2}(D, \bar{D})+\theta_{4} R^{2}(d D, d \bar{D})+\theta_{5}\left(1-\frac{\left|r_{a b}-\overline{r_{a b}}\right|}{\overline{r_{a b}}}\right)\right]
\end{aligned}
$$

where the series $G, D$ and $\bar{G}, \bar{D}$ are weighted geometrically emphasising the latest data points in each phase, $R^{2}$ is the coefficient of determination, $r_{a b}, \overline{r_{a b}}$ are the antibody ratio at the end of the first phase of the simulation and reported, respectively. We obtained $\overline{r_{a b}}$ from serosurveys carried out by ICMR. Our simulation is on a daily basis, $d G, d D, d \bar{G}, d \bar{D}$ are calculated daily. 
medRxiv preprint doi: https://doi.org/10.1101/2022.02.02.22270353; this version posted February 4, 2022. The copyright holder for this preprint (which was not certified by peer review) is the author/funder, who has granted medRxiv a license to display the preprint in perpetuity.

It is made available under a CC-BY-NC-ND 4.0 International license.

Fitting for India
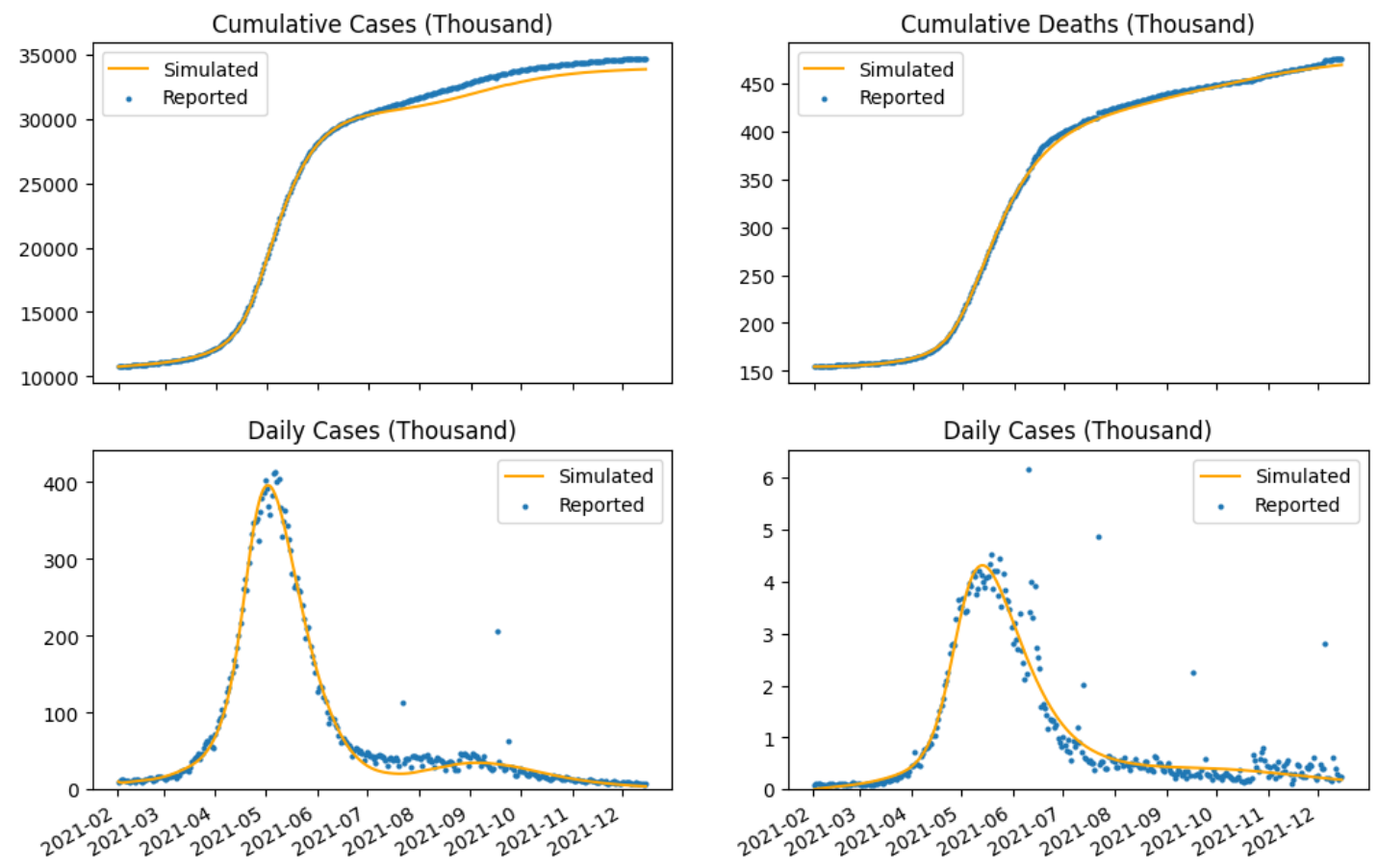

Figure 4: Fitting results for India 
medRxiv preprint doi: https://doi.org/10.1101/2022.02.02.22270353; this version posted February 4, 2022. The copyright holder for this preprint (which was not certified by peer review) is the author/funder, who has granted medRxiv a license to display the preprint in perpetuity.

It is made available under a CC-BY-NC-ND 4.0 International license .

\begin{tabular}{|c|c|c|c|c|c|c|}
\hline State & R2(confirmed) & R2(confirmed phase1) & R2(confirmed phase2) & R2(death) & R2(death phase1) & R2(death phase2) \\
\hline India & 0.9961 & 0.9997 & 0.7951 & 0.9993 & 0.9998 & 0.9750 \\
\hline Andaman and Nicobar & 0.9967 & 0.9962 & 0.5327 & 0.1906 & 0.9662 & -1831.9827 \\
\hline Jharkhand & 0.9998 & 0.9995 & 0.7872 & 0.9927 & 0.9984 & -187.2522 \\
\hline Bihar & 0.9997 & 0.9996 & -0.6258 & 0.0798 & 0.9389 & -63.8096 \\
\hline Ladakh & 0.9941 & 0.9955 & -0.6089 & 0.7665 & 0.9558 & -55.2289 \\
\hline Lakshdweep & 0.9223 & 0.9985 & -8.3217 & 0.7206 & 0.9570 & -35.6842 \\
\hline Tripura & 0.9936 & 0.9985 & 0.9132 & -0.5002 & 0.9864 & -23.2177 \\
\hline Rajesthan & 0.9992 & 0.9988 & -33.4340 & 0.9990 & 0.9996 & -9.8509 \\
\hline Uttar Pradesh & 0.9980 & 0.9998 & -158.6117 & 0.9873 & 0.9934 & -8.8216 \\
\hline Madhya Pradesh & 0.9989 & 0.9997 & -40.8878 & 0.7687 & 0.9852 & -6.0478 \\
\hline Haryana & 0.9994 & 0.9981 & -0.0964 & 0.9795 & 0.9993 & -2.6716 \\
\hline Tamil Nadu & 0.9992 & 0.9976 & 0.9520 & 0.9431 & 0.9974 & -2.1433 \\
\hline Sikkim & 0.9987 & 0.9989 & 0.9882 & 0.8127 & 0.9726 & -1.4990 \\
\hline Chhattisgarh & 0.9996 & 0.9997 & -0.3986 & 0.9994 & 0.9995 & -1.3777 \\
\hline Uttarakhand & 0.9993 & 0.9984 & -0.4632 & 0.9972 & 0.9993 & -0.8500 \\
\hline Odisha & 0.9966 & 0.9998 & 0.8474 & 0.5614 & 0.9976 & -0.8083 \\
\hline Jammu and Kashmir & 0.9941 & 0.9991 & -0.1017 & 0.9954 & 0.9943 & -0.4672 \\
\hline Delhi & 0.9996 & 0.9996 & -4.5287 & 0.9996 & 0.9991 & -0.4389 \\
\hline Chandigarh & 0.9987 & 0.9982 & 0.8754 & 0.9540 & 0.9930 & -0.4292 \\
\hline Andhra Pradesh & 0.9970 & 0.9999 & 0.7953 & 0.9504 & 0.9899 & -0.3936 \\
\hline Goa & 0.9908 & 0.9988 & -0.6514 & 0.9859 & 0.9991 & -0.3780 \\
\hline Nagaland & 0.9974 & 0.9988 & 0.9628 & 0.9327 & 0.9913 & -0.0927 \\
\hline Puducherry & 0.9920 & 0.9977 & 0.2387 & 0.9932 & 0.9957 & -0.0703 \\
\hline Daman and Diu & 0.9996 & 0.9996 & -0.0732 & 0.6843 & 0.6575 & 0.0000 \\
\hline Assam & 0.9379 & 0.9998 & -0.4587 & 0.9603 & 0.9977 & 0.2740 \\
\hline Maharastra & 0.9919 & 0.9991 & 0.2586 & 0.9848 & 0.9975 & 0.5334 \\
\hline West Bengal & 0.9995 & 0.9996 & 0.9377 & 0.9911 & 0.9980 & 0.5844 \\
\hline Manipur & 0.9967 & 0.9972 & 0.9811 & 0.9472 & 0.9714 & 0.6193 \\
\hline Gujarat & 0.9998 & 0.9997 & -1.8440 & 0.9979 & 0.9934 & 0.6432 \\
\hline Telangana & 0.9969 & 0.9995 & 0.7377 & 0.9963 & 0.9982 & 0.7271 \\
\hline Punjab & 0.9989 & 0.9981 & -0.7284 & 0.9992 & 0.9989 & 0.7297 \\
\hline Himachal Pradesh & 0.9886 & 0.9931 & 0.1555 & 0.9966 & 0.9910 & 0.7871 \\
\hline Mizoram & 0.5215 & 0.9979 & 0.0192 & 0.9473 & 0.9899 & 0.8839 \\
\hline Meghalaya & 0.8741 & 0.9937 & 0.0778 & 0.9854 & 0.9900 & 0.8856 \\
\hline Karnataka & 0.9991 & 0.9999 & 0.7588 & 0.9989 & 0.9995 & 0.8907 \\
\hline Arunachal Pradesh & 0.9952 & 0.9980 & 0.9640 & 0.9925 & 0.9725 & 0.9446 \\
\hline Kerala & 0.9633 & 0.9997 & 0.8058 & 0.9825 & 0.9821 & 0.9538 \\
\hline
\end{tabular}

Table 1: $\mathrm{R}^{2}$ score for India and all states

\subsection{Second-Phase Fitting for reopen}

In the second phase when removal of lockdown started we consider release of people from lockdown via a linear function with a starting date in a range after 1 week of release of lockdown, i.e. starting on the 17th of June. The release of population into the susceptible set is assumed to be a linear function again and the rate of release dictates the speed at which the policy restrictions are being relaxed and the behavior of the population.

The growth of the infection curve is strongly dependent on this rate, as we will illustrate in our projections. During this phase we estimate the rate of release, the start of release and the size of the release.

$$
L_{2}\left(h_{R}\right)=-\left[\theta_{1} R^{2}(G, \bar{G})+\theta_{2} R^{2}(d G, d \bar{G})+\theta_{3} R^{2}(D, \bar{D})+\theta_{4} R^{2}(d D, d \bar{D})\right]
$$




\subsection{Fitting parameters}

We illustrate our fitting for a combined first and second phase in Figure 4. The goodness of fit for all Indian states is illustrated in the supplementary material. The $R^{2}$ values illustrating the goodness of fit was determined and listed in Table 1 .

The $R^{2}$ values for the combined fitting, i.e. first and second phase, of the infection case numbers is contained in the first column while the $R^{2}$ values for the combined fitting of the number of deaths is in the 4 th column and consistently high. $R^{2}$ values for individual states are inconsistent in the second phase for both the infected cases and the deaths since each individual state had applied varying policies, some including multiple attempts at restrictive policies including lockdown during this phase.

\subsection{Model based Projections}

The model was used to create projections in order to help guide policy advice and communicated to Dr. Paul, Niti Ayog, of the Indian government.

While successive projections illustrated in Figure 1 had been communicated periodically, we provide details here of the simulation report in December subsequently modified as more data emerged.

Projections including both the delta and the highly transmissible Omicron variant. We utilized vaccination rate efficacy to include breakthrough cases.

The projections on the Sars-Cov2 crisis are based on data until December 15th, 2021. We estimated the transmissibility of Omicron variant from the South African data of the virus and comparing the transmissible factor with the previous variant to compute a beta_factor that was used to adjust the transmission factor for Omicron. The vaccine efficacy is adjusted for and is considered less effective w.r.t. Omicron. We present results that are parameterized on the vaccine efficiency and listed below.

Some key assumptions:

- We provide projections based on case analysis where the efficacy of the vaccine against the Omicron variant is reduced by $50 \%$ and $25 \%$

- We assume that there is a seeding of 50 cases per state on December 15st, 2021

- We provide two scenarios, one where there will be (i) no subsequent lockdowns and (ii) there will be mild lockdowns, impacting the social distancing and the susceptible population by a factor of 15,20 and $25 \%$.

\section{Parameters}

As in prior reports, we establish projections that are based on the initial growth in population that were part of transmission dynamics in the early part of 2021 and that provides a baseline for rise in cases. After lockdown removal in June, a release of population that adopts the behaviour of the baseline is estimated. Using the baseline scenario we quantify the second release with respect to the susceptible population estimation that was computed during February-March 2021 when restrictions were removed in late January.

- Baseline established during February to March, 2021. 

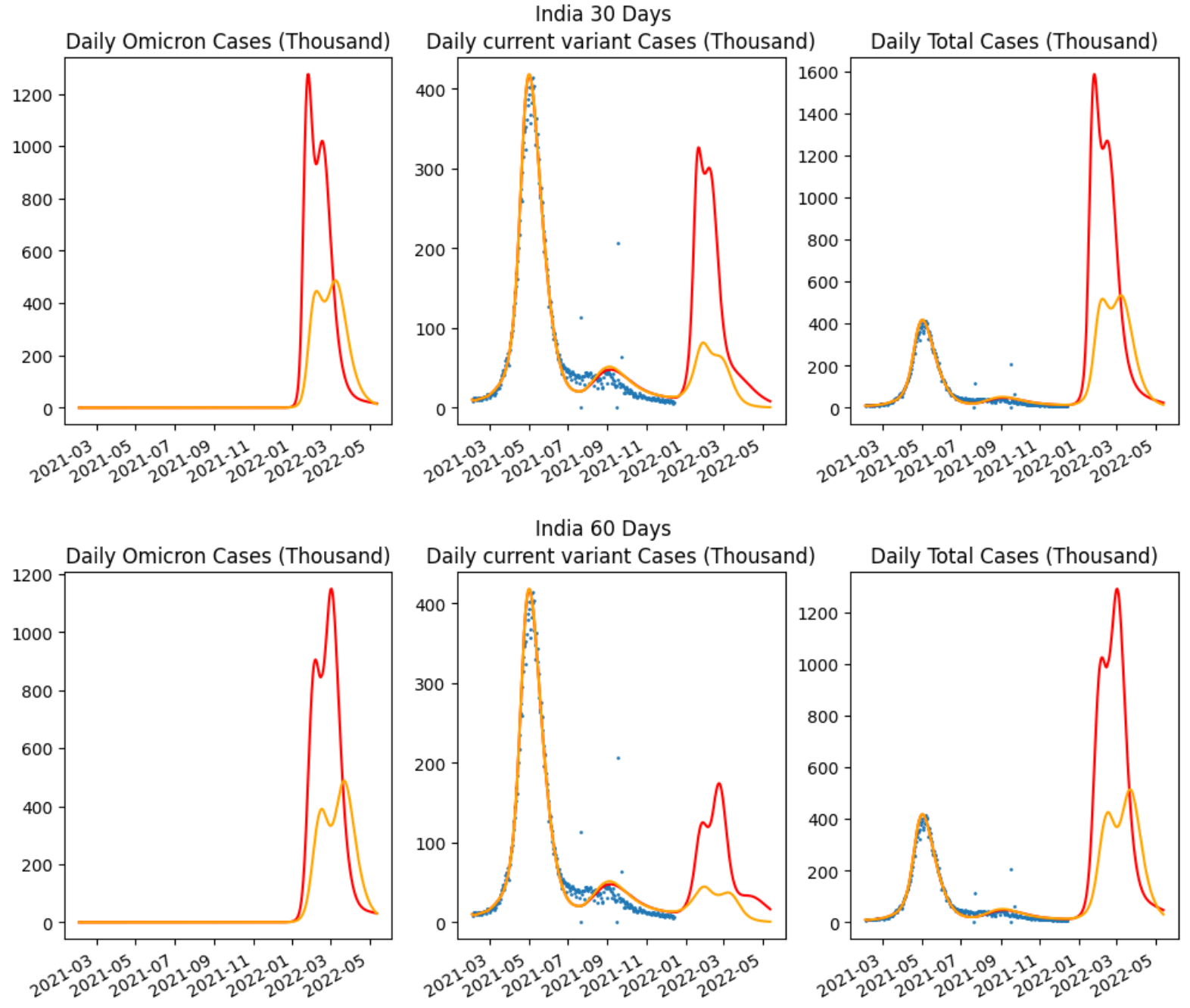

Figure 5: 50\% Vaccine efficacy against Omicron, No lockdown: Comparison of two scenarios: releasing population having occurred from lockdown illustrating two release rates, high (30 days) and moderate (60 days) Red indicates the worst case behavior (Scenario 1) and Orange indicate the optimistic Scenario 2. The leftmost graph in each row is for the projected cases of the omicron variant whereas the third graph is for the projected confirmed cases of the two variants together

- Average Vaccination rates: 3.0M (30 Lakhs)/day or approximately 0.225\%/day till August 16th.

- Current rates averaged to $5 \mathrm{M}$ vaccinations/day.

Projected vaccination for further releases from lockdown is averaged to approximately $8 \mathrm{M}$ vaccinations/day for first dose.

- Current additional lockdown removal date: December 16th, 2021 and lockdown release rate are $100 \%$ of baseline over 30 days (high release) and 60 days (low release) given the lack of social 

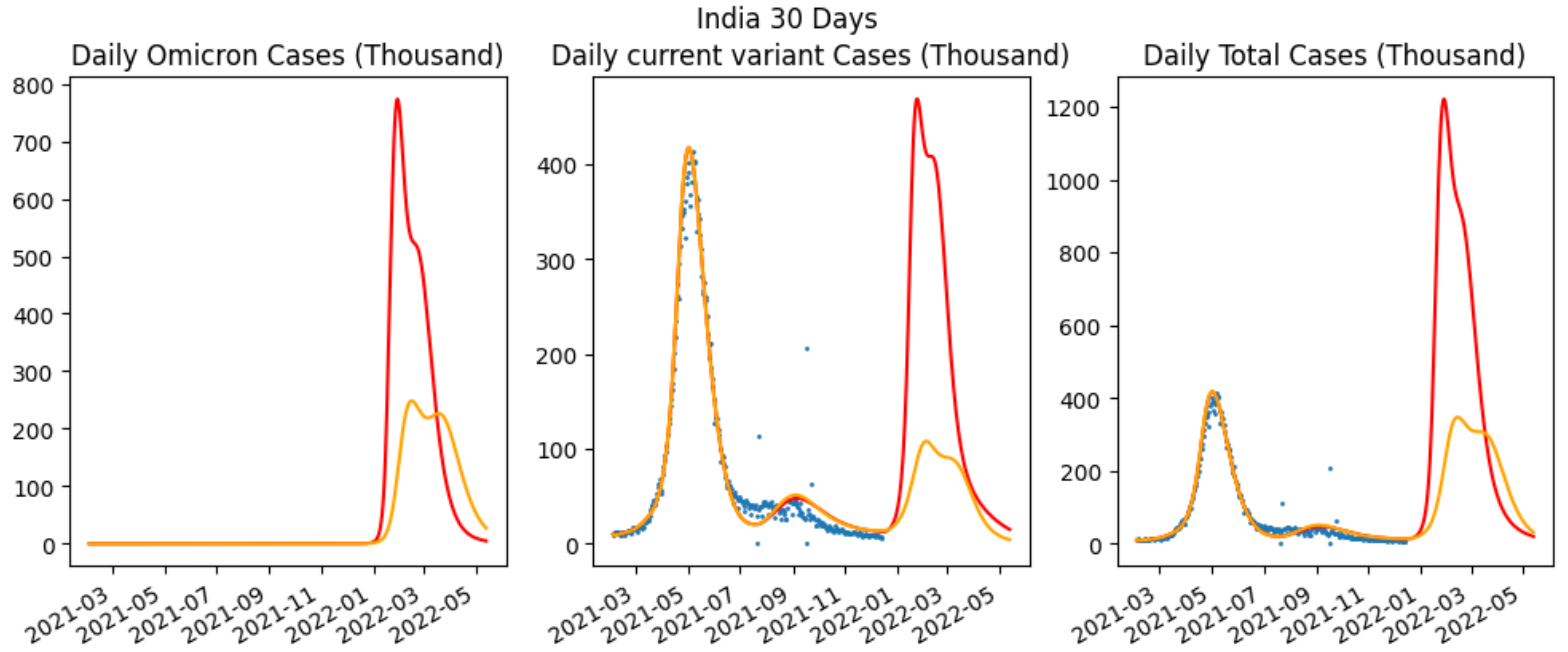

Daily Omicron Cases (Thousand)
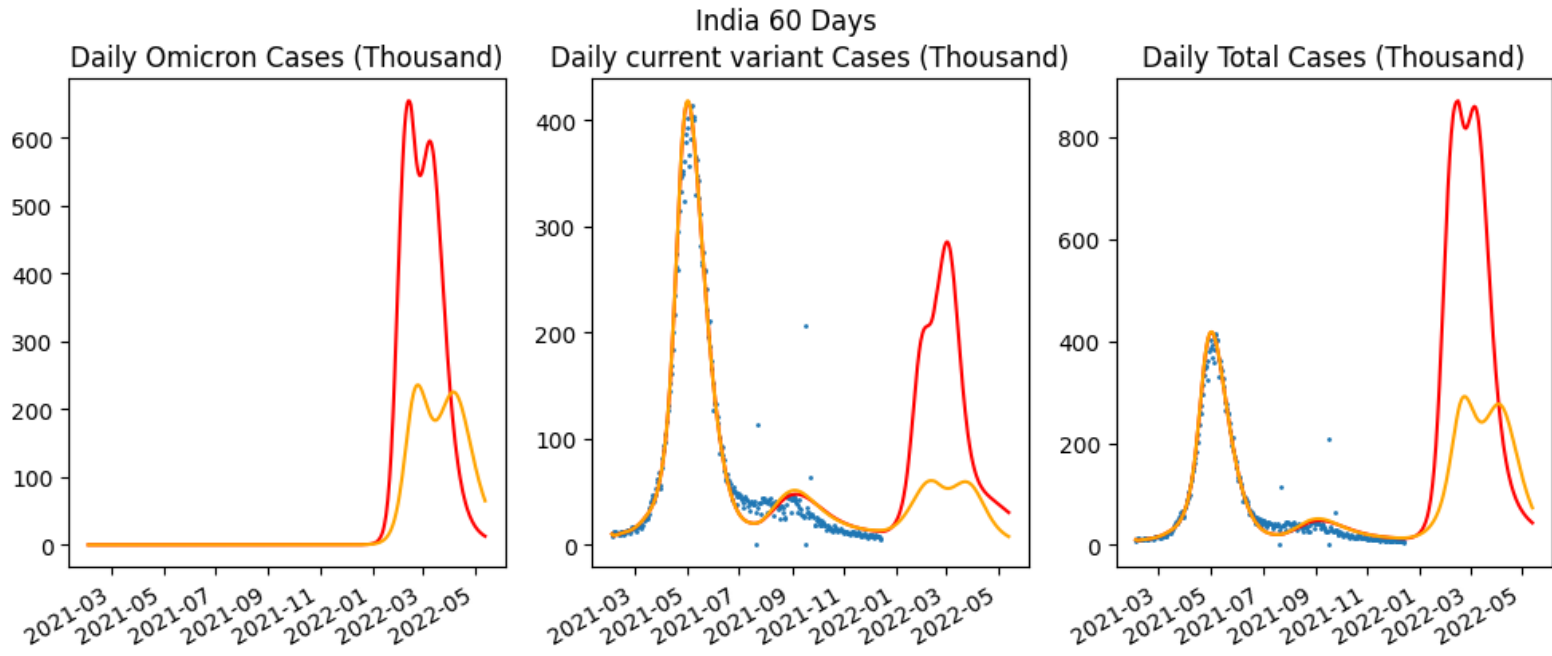

Figure 6: 75\% Vaccine efficacy against Omicron, No lockdown: Comparison of two scenarios: releasing population having occurred from lockdown with high release (30 days) and low release (60 days). Red indicates the worst case behavior (Scenario 1) and Orange indicate the optimistic Scenario 2. The leftmost graph in each row is for the projected cases of the omicron variant whereas the third graph is for the projected confirmed cases of the two variants together

distancing during the months of November and December.

- We also consider projections based on partial lockdown which we estimate to be $10 \%$.

- We model the loss of efficacy of vaccines as reduced linearly over 4 months by $50 \%$. This loss is causing breakthrough infections.

- We model the efficacy of vaccines against Omicron as $50 \%$ and $75 \%$ of the original efficacy. 

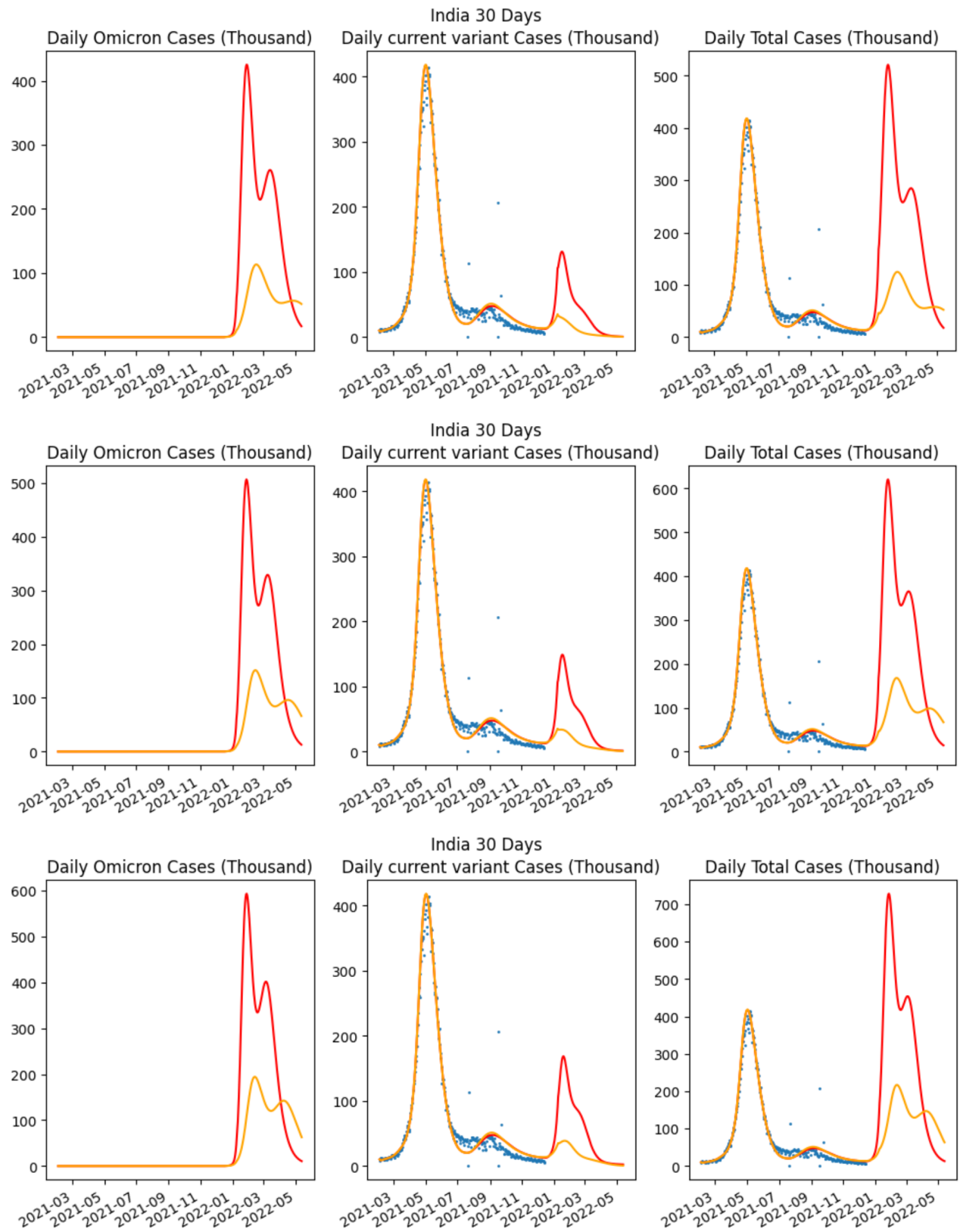

Figure 7: 50\% Vaccine efficacy against Omicron, Lockdown Cases, 25,20 and $15 \%$ starting January 8th: Comparison of three scenarios: releasing population from lockdown over 30,60 and 90 days. Red indicates the worst case behavior (Scenario 1) and Orange indicate the optimistic Scenario 2. The leftmost graph in each row is for the projected cases of the omicron variant whereas the third graph is for the projected confirmed cases of the two variants together 


\section{Discussion}

The modeling shows projections (Figure 5) that were concerning, especially due to reduction in social distancing countered by reported milder symptoms of the new variant. We model this release in two scenarios where the susceptible population is increasing at a substantial rate, the rate being represented by a release of the base population of 30,60 days.

We consider two cases below.

\subsubsection{No Lockdown assumption:}

The two scenarios considered as in the previous report are provided below and evaluated in December, 2021. The indicated peaks are substantial.

Scenario 1 (pessimistic scenario-red curve): The pessimistic scenario is when the next release is estimated from the established baseline scenario. The population that has already become part of the susceptible pool after the relaxation from lockdown is ignored.

This results indicate a substantial rise in infections when rules regarding lockdown are relaxed. Our results project that there would be increasing high case loads during January and February, extending into March and April months. It is critical that utmost care be taken to ensure social distancing and mask usage, reduced capacity of interior locations and marketplaces, especially in restaurants and bars [19]. We are observing peak infection cases in the peaking at around 1.6 Million cases per day for $50 \%$ efficacy loss and 1.2 Million cases for $25 \%$ efficacy loss.

Further peaks could be progressively delayed and limited in size if social distancing measure are strictly followed. Note that this does not account for any variants that can breakthrough the vaccine defense. While this appears to be an obvious statement, the second wave of infections illustrate the impact of lack of continued emphasis on these measures.

Scenario 2 (optimistic scenario-orange curve): This scenario assumes that the susceptible that are added after relaxation of lockdown, but before 15th December, 2021, were already exhibiting the baseline behaviour and are discounted from future release of population into the susceptible pool. This is an optimistic scenario.

These two scenarios are illustrated in Figure 5 and Figure 6

\subsubsection{Lockdown assumption:}

We also consider the situation when a mild lockdown is implemented on January 8th. This lockdown is assumed to reduce 15,20 and $25 \%$ of the susceptible population for the high release scenario prior to the date of lockdown.

We observe substantial benefits due to partial lockdown, being limited to less than half of the cases at peak when no lockdown is enforced ((Figure 7p), which would have resulted in substantial cases and the resulting hospitalization would have hampered the health system. 


\section{Conclusions}

Early warning systems which project long range behavior more than a month in advance, while not pointing exact peaks and case projections are very useful for policy decisions.

We also experimented with short term models that relied on initial growth rates. These model did not fully capture the gravity of the situation even when executed a month in advance, primarily due to mixed data from the virus variants.

At the end of January, 2022 we observe that due to the mild symptoms there is considerable lack of reporting of cases and infection numbers are no longer a good measure of the disease.

We would like to thank Dr. V.K. Paul and Professor S.N. Maheshwari (Emeritus, IIT Delhi) for their suggestions and help during the development of these projections.

\section{References and Notes}

[1] Cleo Anastassopoulou, Lucia Russo, Athanasios Tsakris, and Constantinos Siettos. Data-based analysis, modelling and forecasting of the COVID-19 outbreak. PloS one, 15(3):e0230405, March 2020 .

[2] Sercan Arik, Chun-Liang Li, Jinsung Yoon, Rajarishi Sinha, Arkady Epshteyn, Long T. Le, Vikas Menon, and Shashank Singh. Interpretable Sequence Learning for COVID-19 Forecasting. Available at https://storage.googleapis.com/covid-external/COVID-19ForecastWhitePaper.pdf.

[3] David W Berger, Kyle F Herkenhoff, and Simon Mongey. An SEIR Infectious Disease Model with Testing and Conditional Quarantine. Working Paper 26901, National Bureau of Economic Research, March 2020.

[4] I De Falco, A Della Cioppa, U Scafuri, and E Tarantino. Coronavirus covid-19 spreading in italy: optimizing an epidemiological model with dynamic social distancing through differential evolution. arXiv preprint arXiv:2004.00553, 2020.

[5] Herbert W Hethcote. The basic epidemiology models: models, expressions for R0, parameter estimation, and applications. In Mathematical understanding of infectious disease dynamics, pages 1-61. World Scientific, 2009.

[6] William Ogilvy Kermack and Anderson G McKendrick. A contribution to the mathematical theory of epidemics. Proceedings of the Royal Society of London. Series A, Containing papers of a mathematical and physical character, 115(772):700-721, 1927.

[7] William Ogilvy Kermack and Anderson G McKendrick. "contributions to the mathematical theory of epidemics. ii.- - the problem of endemicity". Proceedings of the Royal Society of London. Series A, Containing papers of a mathematical and physical character, 138(834):55-83, 1932.

[8] William Ogilvy Kermack and Anderson G McKendrick. Contributions to the mathematical theory of epidemics. III.-Further studies of the problem of endemicity. Proceedings of the Royal Society of London. Series A, Containing Papers of a Mathematical and Physical Character, 141(843):94-122, 1933. 
[9] Stephen M Kissler, Christine Tedijanto, Edward Goldstein, Yonatan H Grad, and Marc Lipsitch. Projecting the transmission dynamics of sars-cov-2 through the postpandemic period. Science, 368(6493):860-868, 2020.

[10] Adam J Kucharski, Timothy W Russell, Charlie Diamond, Yang Liu, John Edmunds, Sebastian Funk, Rosalind M Eggo, Fiona Sun, Mark Jit, James D Munday, et al. Early dynamics of transmission and control of COVID-19: a mathematical modelling study. The Lancet Infectious Diseases, 20:553-558, May 2020.

[11] Li, Ruiyun and Pei, Sen and Chen, Bin and Song, Yimeng and Zhang, Tao and Yang, Wan and Shaman, Jeffrey. Substantial undocumented infection facilitates the rapid dissemination of novel coronavirus (SARS-CoV-2). Science, 368(6490):489-493, 2020.

[12] Clément Massonnaud, Jonathan Roux, and Pascal Crépey. COVID-19: Forecasting short term hospital needs in France. medRxiv, doi: https://doi.org/10.1101/2020.03.16.20036939, March 2020.

[13] K Roosa, Y Lee, R Luo, A Kirpich, R Rothenberg, JM Hyman, P Yan, and G Chowell. Real-time forecasts of the COVID-19 epidemic in China from February 5th to February 24th, 2020. Infectious Disease Modelling, 5:256-263, 2020.

[14] Kimberlyn Roosa, Yiseul Lee, Ruiyan Luo, Alexander Kirpich, Richard Rothenberg, James M Hyman, Ping Yan, and Gerardo Chowell. Short-term forecasts of the COVID-19 epidemic in Guangdong and Zhejiang, China: February 13-23, 2020. Journal of Clinical Medicine, 9(2):596, 2020.

[15] Thomas C Tsai, Benjamin H Jacobson, and Ashish K Jha. American hospital capacity and projected need for COVID-19 patient care. Health Affairs Blog, doi: 10.1377/hblog20200317.457910, March 2020 .

[16] Joseph $\mathrm{T} \mathrm{Wu}$, Kathy Leung, and Gabriel M Leung. Nowcasting and forecasting the potential domestic and international spread of the 2019-nCoV outbreak originating in Wuhan, China: a modelling study. The Lancet, 395(10225):689-697, 2020.

[17] Bo Xu, Bernardo Gutierrez, Sumiko Mekaru, Kara Sewalk, Lauren Goodwin, Alyssa Loskill, Emily L Cohn, Yulin Hswen, Sarah C Hill, Maria M Cobo, et al. Epidemiological data from the COVID-19 outbreak, real-time case information. Scientific data, 7(1):1-6, 2020.

[18] Juanjuan Zhang, Maria Litvinova, Yuxia Liang, Yan Wang, Wei Wang, Shanlu Zhao, Qianhui Wu, Stefano Merler, Cécile Viboud, Alessandro Vespignani, Marco Ajelli, and Hongjie Yu. Changes in contact patterns shape the dynamics of the covid-19 outbreak in china. Science, 368(6498):14811486, 2020.

[19] Yi Zhang and Sanjiv Kapoor. Hidden Parameters Impacting Resurgence of SARS-CoV-2 Pandemic. medRxiv, 2021. 